\title{
STOCHASTIC MECHANICS AND QUANTUM INTERFERENCE
}

\author{
N. CUFARO PETRONI \\ Dipartimento di Fisica dell'Università and INFN, Bari, Italy
}

Received 15 August 1989; accepted for publication 15 September 1989

Communicated by J.P. Vigier

\begin{abstract}
It is shown that in the framework of the stochastic interpretation of quantum mechanics it is always possible to determine correct real positive transition probabilities that can be added together to obtain the quantum interference patterns. The implication of this fact on the possibility that quantum micro-objects follow trajectories in space and time is discussed. A derivation is given of a path integral formula yielding these transition probabilities.
\end{abstract}

It is usual to say that quantum mechanics rules out the possibility of speaking of particle trajectories in space and time. A way to show this is to remark that, as recently confirmed [1] (and contrary to classical particles) quantum micro-objects individually interfere in a two-slit experiment: a fact that immediately raises the question (negatively answered in the usual Copenhagen interpretation) if particles travel, or not, in real space and time. Since there is today a number of theories [2,3], inherently based on the idea of particle trajectories, which extensively simulate the quantum results, all this discussion must be up-dated. In order to do this, in the framework of one of these theories [3], let us briefly summarize the origin of the Copenhagen no go statement.

If a quantum system obeys the Schrödinger equation

$\mathrm{i} \hbar \partial_{t} \psi(\boldsymbol{r}, t)=\left(-\frac{\hbar^{2}}{2 m} \nabla^{2}+V(\boldsymbol{r}, t)\right) \psi(\boldsymbol{r}, t)$

and if $K\left(\boldsymbol{r}, t ; \boldsymbol{r}^{\prime}, t^{\prime}\right)$ with $t>t^{\prime}$ denotes the corresponding Green function, we know that the wavefunction

$\psi(\boldsymbol{r}, t)=\int_{R^{3}} K\left(\boldsymbol{r}, t ; \boldsymbol{r}^{\prime}, t_{\mathrm{i}}\right) \psi_{\mathrm{i}}\left(\boldsymbol{r}^{\prime}, t_{\mathrm{i}}\right) \mathrm{d}^{3} \boldsymbol{r}^{\prime}$

represents the solution of (1) with the initial condition $\psi\left(\boldsymbol{r}, t_{\mathrm{i}}\right)=\psi_{\mathrm{i}}(\boldsymbol{r})$. The kernel $K\left(\boldsymbol{r}, t ; \boldsymbol{r}_{\mathrm{i}}, t_{\mathrm{i}}\right)$ then denotes a solution of (1) corresponding to the initial condition

$\lim _{t, t_{\mathrm{i}}} \psi(\boldsymbol{r}, t)=\delta^{3}\left(\boldsymbol{r}-\boldsymbol{r}_{\mathrm{i}}\right)$,

while $\left|K\left(\boldsymbol{r}, t ; \boldsymbol{r}_{\mathrm{i}}, t_{\mathrm{i}}\right)\right|^{2}$ represents the probability density at the time $t$ under the condition in the sense stated by (3), that the particle was in $r_{\mathrm{i}}$ at the time $t_{\mathrm{i}}$. The functions $K$ evidently satisfy the relations $\left(t_{\mathrm{f}}>t>t_{\mathrm{i}}\right)$

$K\left(\boldsymbol{r}_{\mathrm{f}}, t_{\mathrm{f}} ; \boldsymbol{r}_{\mathrm{i}}, t_{\mathrm{i}}\right)=\int_{\mathrm{R}^{3}} K\left(\boldsymbol{r}_{\mathrm{f}}, t_{\mathrm{f}} ; \boldsymbol{r}, t\right) K\left(\boldsymbol{r}, t ; \boldsymbol{r}_{\mathrm{i}}, t_{\mathrm{i}}\right) \mathrm{d}^{3} r$.

Now from relations (2) and (4) it immediately follows that in general

$|\psi(\boldsymbol{r}, t)|^{2} \neq \int_{\mathbb{R}^{3}}\left|K\left(\boldsymbol{r}, t ; \boldsymbol{r}^{\prime}, t^{\prime}\right)\right|^{2}\left|\psi_{\mathrm{i}}\left(\boldsymbol{r}^{\prime}\right)\right|^{2} \mathrm{~d}^{3} \boldsymbol{r}^{\prime}$, 
$\left|K\left(\boldsymbol{r}_{\mathrm{f}}, t_{\mathrm{f}} ; \boldsymbol{r}_{\mathrm{i}}, t_{\mathrm{i}}\right)\right|^{2} \neq \int_{\mathbb{R}^{3}}\left|K\left(\boldsymbol{r}_{\mathrm{f}}, t_{\mathrm{f}} ; \boldsymbol{r}, t\right)\right|^{2}\left|K\left(\boldsymbol{r}, t ; \boldsymbol{r}_{\mathrm{i}}, t_{\mathrm{i}}\right)\right|^{2} \mathrm{~d}^{3} \boldsymbol{r}$.

Let us now compare relations (5) and (6) with the classical relations

$\rho(\boldsymbol{r}, t)=\int_{\mathrm{R}^{3}} P\left(\boldsymbol{r}, t ; \boldsymbol{r}^{\prime}, t_{\mathrm{i}}\right) \rho_{\mathrm{i}}\left(\boldsymbol{r}^{\prime}\right) \mathrm{d}^{3} \boldsymbol{r}^{\prime}$,

$P\left(\boldsymbol{r}_{\mathrm{f}}, t_{\mathrm{f}} ; \boldsymbol{r}_{\mathrm{i}}, t_{\mathrm{i}}\right)=\int_{\mathrm{R}^{3}} P\left(\boldsymbol{r}_{\mathrm{f}}, t_{\mathrm{f}} ; \boldsymbol{r}, t\right) P\left(\boldsymbol{r}, t ; \boldsymbol{r}_{\mathrm{i}}, t_{\mathrm{i}}\right) \mathrm{d}^{3} \boldsymbol{r}$,

where $\rho$ and $P$ are respectively the probability density and the transition probability density of a Markov process; namely the solutions of a Fokker-Planck equation with the initial conditions

$\rho\left(\boldsymbol{r}, t_{\mathrm{i}}\right)=\rho_{\mathrm{i}}(\boldsymbol{r})$,

$\lim _{t \downarrow t_{\mathrm{i}}} P\left(\boldsymbol{r}, t ; \boldsymbol{r}_{\mathrm{i}}, t_{\mathrm{i}}\right)=\delta^{3}\left(\boldsymbol{r}-\boldsymbol{r}_{\mathrm{i}}\right)$.

Since these classical equations are seen as an expression of the idea that a Markov process, going from $\boldsymbol{r}_{\mathrm{i}}$ to $r_{\mathrm{f}}$, must pass through some of the possible intermediate positions at the intermediate times, the relations (5) and (6) are generally considered as a form of the opposite statement i.e. in quantum mechanics only the complex amplitudes can always be added, as in (2) and (4), but not, in general, the probabilities. More precisely, if a particle travels from $\boldsymbol{r}_{\mathrm{i}}$ to $\boldsymbol{r}_{\mathrm{f}}$, we cannot think of it as being somewhere, with a given probability, at intermediate times, unless we verify our statements by measuring the intermediate positions. Indeed since we know that such measurements would change the state of our system (so that (7) and (8) will be verified) a number of quantum effects, like interferences, will be lost. It is clear that in this situation it is very difficult to speak of a trajectory between $\boldsymbol{r}_{\mathrm{i}}$ and $\boldsymbol{r}_{\mathrm{f}}$.

Let us illustrate these ideas by means of a simplified two-slit experiment, i.e., suppose we have a screen, with two holes located in $z_{1}$ and $z_{2}$, between a source of particles and a detector in $r$, and ask for the probability density of the detected particles in the following three situations:

(1) hole 1 open, hole 2 closed;

(2) hole 1 closed, hole 2 open;

(3) hole 1 open, hole 2 open.

From the Green function of the free Schrödinger equation [4] we have

$K_{0}\left(\boldsymbol{r}, t ; \boldsymbol{r}^{\prime}, t^{\prime}\right)=\left(\frac{m}{2 \pi \mathrm{i} \hbar\left(t-t^{\prime}\right)}\right)^{3 / 2} \exp \left(\frac{\mathrm{i} m\left(\boldsymbol{r}-\boldsymbol{r}^{\prime}\right)^{2}}{\bar{h}} \overline{2\left(t-t^{\prime}\right)}\right)$

and we can calculate the respective wave functions:

$\psi^{(1)}(\boldsymbol{r}, t)=K_{0}\left(\boldsymbol{r}, t ; \boldsymbol{r}_{1}, t_{\mathrm{i}}\right), \quad \psi^{(2)}(\boldsymbol{r}, t)=K_{0}\left(\boldsymbol{r}, t ; \boldsymbol{r}_{2}, t_{\mathrm{i}}\right)$,

$\psi^{(1,2)}(\boldsymbol{r}, t)=C_{1} K_{0}\left(\boldsymbol{r}, t ; \boldsymbol{r}_{1} t_{\mathrm{i}}\right)+C_{2} K_{0}\left(\boldsymbol{r}, t ; \boldsymbol{r}_{2}, t_{\mathrm{i}}\right)$,

where $\left|C_{1}\right|^{2}+\left|C_{2}\right|^{2}=1$, and verify that the probabilities do not add, i.e. that

$\left|\psi^{(1,2)}(r, t)\right|^{2} \neq\left|C_{1}\right|^{2}\left|\psi^{(1)}(r, t)\right|^{2}+\left|C_{2}\right|^{2}$,

the difference being in the interference terms. On the other hand, in a classical situation, characterized by the fact that a particle is always coming from one of the two holes even when they are both open, from (7) and since

$\rho^{(1)}(\boldsymbol{r})=\delta^{3}\left(\boldsymbol{r}-\boldsymbol{r}_{1}\right), \quad \rho^{(2)}(\boldsymbol{r})=\delta^{3}\left(\boldsymbol{r}-\boldsymbol{r}_{2}\right)$,

$\rho_{i}^{(1,2)}(r)=a_{1} \delta^{3}\left(r-r_{1}\right)+a_{2} \delta^{3}\left(r-r_{2}\right)$ 
where $a_{1}+a_{2}=1, a_{1}>0, a_{2}>0$, we would have

$\rho^{(1)}(\boldsymbol{r}, t)=P\left(\boldsymbol{r}, t ; \boldsymbol{r}_{1}, t_{\mathrm{i}}\right), \quad \rho^{(2)}(\boldsymbol{r}, t)=P\left(\boldsymbol{r}, t ; \boldsymbol{r}_{2}, t_{\mathrm{i}}\right)$,

$\rho^{(1,2)}(\boldsymbol{r}, t)=a_{1} P\left(\boldsymbol{r}, t ; r_{1}, t_{\mathrm{i}}\right)+a_{2} P\left(\boldsymbol{r}, t ; \boldsymbol{r}_{2}, t_{\mathrm{i}}\right)=a_{1} \rho^{(1)}(\boldsymbol{r}, t)+a_{2} \rho^{(2)}(\boldsymbol{r}, t)$,

so that probabilities would add. The striking contrast between the two descriptions of the case (1), (2) is the basis of the quantum mechanical rejection of the idea that particles come either from one hole or from the other and, by extension, of the idea that particles follow non-directly observed paths when they travel from $\boldsymbol{r}_{\mathrm{i}}$ to $r_{\mathrm{f}}$. As a consequence Feynman's formula giving the Green functions as a path integral [4], namely

$K\left(\boldsymbol{r}_{\mathrm{f}}, t_{\mathrm{f}} ; \boldsymbol{r}_{\mathrm{i}}, t_{\mathrm{i}}\right)=\int_{\mathrm{i} \rightarrow \mathrm{f}} \exp \left(\frac{\mathrm{i}}{\hbar} \int_{t_{\mathrm{i}}}^{t_{\mathrm{f}}} \mathscr{L}[\mathrm{d} \boldsymbol{r}(t) / \mathrm{d} t, \boldsymbol{r}(t), t] \mathrm{d} t\right) \mathrm{D}[\boldsymbol{r}()]$,

with the usual meaning of the symbols, is only a formal addition of complex amplitudes and not the sum of probabilities for paths and hence cannot be derived along a true probabilistic line of reasoning.

Since we now know that stochastic mechanics [3], i.e. a theory based on the idea that particles follow random trajectories in space and time, can simulate all the known quantum results by means of stochastic processes $\xi(t)$ which are solutions of stochastic differential equations of the form

$\mathrm{d} \xi(t)=v_{(+)}(\xi(t), t) \mathrm{d} t+\mathrm{d} \boldsymbol{\beta}(t)$,

where $\boldsymbol{\beta}(t)$ is a Wiener process (with diffusion constant $\nu=\hbar / 2 m$ ) it is important to point out that there are two different interpretational schemes of (18) leading to completely different results [5]:

(I) We can consider $\boldsymbol{v}_{(+)}$as a given field and $\boldsymbol{\xi}(t)$ as a complete description of the state of the system. In this case, which is a stochastic generalization of classical mechanics, we obtain Langevin-type equations and a dissipative dynamics that cannot reproduce the quantum effects.

(II) We can assume that $\xi(t)$ does not contain all the information about the state of our system, but is only a sort of configurational variable, the rest of the information being stored in the form of $v_{(+)}$which now is not given, but plays the role of an additional dynamical variable whose values must be determined by means of a variational principle [2]. As we shall now show, this case, which corresponds to stochastic control theory [6], leads directly, by means of a suitable choice of the Lagrangian, to a perfect reproduction of the quantum results.

In this stochastic interpretation, to a quantum system described by (1) we can associate an entire family of processes, one for every state, in the following way: we fix a solution of (1) and then we calculate

$v_{(+)}(r, t)=2 \nu \nabla W_{(+)}(r, t)$,

where

$W_{(+)}(\boldsymbol{r}, t)=\ln \{R(\boldsymbol{r}, t) \exp [S(\boldsymbol{r}, t) / \hbar]\}, \quad \psi(\boldsymbol{r}, t)=R(\boldsymbol{r}, t) \exp [\mathrm{i} S(\boldsymbol{r}, t) / \hbar]$,

which determines the form of (18). Then we fix the initial condition

$\boldsymbol{\xi}\left(t_{\mathrm{i}}\right)=\boldsymbol{\xi}_{\mathrm{i}}$,

by determining the random variable $\xi_{\mathrm{i}}$ from the initial probability density $\left|\psi\left(r, t_{\mathrm{i}}\right)\right|^{2}$. The unique solution [7] of (20) and (21) will be the process associated to our quantum system in the state $\psi$. Of course a different $\psi$ will determine a different process. Note that, since any initial wave function $\psi_{\mathrm{i}}(\boldsymbol{r})$ determines $\psi(\boldsymbol{r}, t), t>t_{\mathrm{i}}$, through (1), we can say that the knowledge of $\psi_{i}(\boldsymbol{r})$ will be enough to determine the process $\boldsymbol{\xi}(t)$.

The dependence of the process $\xi(t)$ on the state is also apparent from the remark that, if through (20) we separate (1) into

$\partial_{\iota} R^{2}+\nabla\left(R^{2} \nabla S / m\right)=0$, 
$\partial_{i} S+\frac{m}{2}\left(\frac{\nabla S}{m}\right)^{2}-\frac{\hbar^{2}}{2 m} \frac{\nabla^{2} R}{R}+V=0$

and then we cast the continuity equation (22) in the form of a forward Fokker-Planck equation for $\rho=R^{2}$, and $\boldsymbol{v}_{(+)}$given in (19), namely

$\partial_{t} \rho=-\nabla\left(\rho v_{(+)}\right)+\nu \nabla^{2} \rho$,

we note that $v_{(+)}$, and hence the form of relation (24), depends on the solution $\rho$, and actually on the complete wave function $\psi$. Hence also the transition probability densities $P\left(\boldsymbol{r}, t ; \boldsymbol{r}^{\prime}, t^{\prime}\right)$, which are solutions of (24) with the initial condition (11), will also depend on the choice of the state $\psi$. Note that, for a given $\psi, \rho$ and $P$ are different solutions of the same (24) corresponding to the initial conditions (10) and (11). Hence, while $\rho$ coincides with $|\psi|^{2}, P$ does not [2].

Let us now rediscuss the two-slit experiment in the light of these remarks. From the wave functions (13) we determine the stochastic processes and the transition probability densities corresponding to every situation by solving (24) with the appropriate form of $\boldsymbol{v}_{(+)}$calculated from (19). We thus get $P^{(1)}\left(\boldsymbol{r}, t ; \boldsymbol{r}_{1}, t_{1}\right) P^{(2)}(\boldsymbol{r}$, $\left.t ; \boldsymbol{r}_{2}, t_{\mathrm{i}}\right)$ and, in the case $(1,2), P^{(1,2)}\left(\boldsymbol{r}, t ; \boldsymbol{r}_{1}, t_{\mathrm{i}}\right)$ and $P^{(1,2)}\left(\boldsymbol{r}, t ; \boldsymbol{r}_{2}, t_{\mathrm{i}}\right) \neq P^{(1,2)}\left(\boldsymbol{r}, t ; \boldsymbol{r}_{1}, t_{\mathrm{i}}\right)$; but of course we should bear in mind that now $P^{(1)}\left(r, t ; r_{1}, t_{1}\right) \neq P^{(1,2)}\left(r, t ; r_{1}, t_{\mathrm{i}}\right)$ and $P^{(2)}\left(\boldsymbol{r}, t ; \boldsymbol{r}_{2}, t_{\mathrm{i}}\right) \neq P^{(1,2)}\left(\boldsymbol{r}, t ; \boldsymbol{r}_{2}, t_{\mathrm{i}}\right)$ since they are derived, in different physical situations, from different wave functions. Moreover the fact that stochastic mechanics reproduces the quantum results means that

$$
\begin{aligned}
& \left|\psi^{(1)}(\boldsymbol{r}, t)\right|^{2}=P^{(1)}\left(\boldsymbol{r}, t ; \boldsymbol{r}_{1}, t_{\mathrm{i}}\right), \quad\left|\psi^{(2)}(\boldsymbol{r}, t)\right|^{2}=P^{(2)}\left(\boldsymbol{r}, t ; \boldsymbol{r}_{2}, t_{\mathrm{i}}\right), \\
& \left|\psi^{(1,2)}(\boldsymbol{r}, t)\right|^{2}=\left|C_{1}\right|^{2} P^{(1,2)}\left(\boldsymbol{r}, t ; \boldsymbol{r}_{1}, t_{\mathrm{i}}\right)+\left|C_{2}\right|^{2} P^{(1,2)}\left(\boldsymbol{r}, t ; \boldsymbol{r}_{2}, t_{\mathrm{i}}\right),
\end{aligned}
$$

because our P's are classical probabilities and satisfy relations (7) and (8). The interference pattern is thus the sum of the appropriate transition probability densities from slit (1) and slit (2). The important point is that in $(25) P^{(1,2)}\left(\boldsymbol{r}, t ; \boldsymbol{r}_{1}, t_{\mathrm{i}}\right)$ and $P^{(1,2)}\left(\boldsymbol{r}, t ; \boldsymbol{r}_{2}, t_{\mathrm{i}}\right)$ are no more the square modulus of a wave function. Of course here the interference effects can be seen as incorporated in the form of the $v_{(+)}$deduced from $\psi^{(1,2)}(r$, $t$ ). In other words stochastic mechanics points out the fact that we can always calculate suitable conditional probabilities that cannot be calculated in the usual quantum formalism: a fact that will be discussed in a subsequent Letter.

In order to stress once more the difference between the two points of view, we will show now how, in a stochastic mechanical context, we can calculate all the transition probability densities by means of path integrals. We will thus get an expression similar to (17), but now it will be obtained by superposing real trajectories, in a true probabilistic derivation, so that in general there will be no corresponding quantum calculation made by means of the squaring of a wave function.

To show this let us first remember some well known results $[7,8]$ of the theory of the stochastic processes concerning the attribution of a probability measure to a trajectory space. By definition if $\eta(t), t \in \mathrm{T}=\left[t_{\mathrm{i}}, t_{\mathrm{f}}\right)$, is a process on a probability space $(\Omega, \mathscr{J}, P)$, the set of its trajectories is the set $R^{3 \mathrm{~T}}$ of all functions $r(): T \rightarrow \mathbb{R}^{3}$. If $\tau=\left\{t_{1}, \ldots, t_{n}\right\}, a=\mathrm{A}_{1} \times \ldots \times \mathrm{A}_{n}$ with $t_{K} \in\left[t_{\mathrm{i}}, t_{\mathrm{f}}\right], \mathrm{A}_{K} \in \mathscr{B}\left(\mathbb{R}^{3}\right)$ for $K=1, \ldots, n$ and

$\mathrm{C}_{t}(\mathrm{~A})=\{\boldsymbol{r}(): \boldsymbol{r}(t) \in \mathrm{A}\} ; \quad t \in \mathbb{R}, \mathrm{A} \in \mathscr{B}\left(\mathbb{R}^{3}\right)$,

the cylinder sets with an $n$-dimensional basis

$\mathrm{C}_{\tau}(a)=\bigcap_{K=1}^{n} \mathrm{C}_{t K}\left(\mathrm{~A}_{K}\right)$

are the subsets of $\mathrm{R}^{3 \mathrm{~T}}$ containing all the trajectories going through the "three-dimensional windows" $\mathrm{A}_{K}$ at times $t_{K}$. Then if $\mathscr{B}\left(\mathrm{R}^{3 \mathrm{~T}}\right)=\sigma(\mathscr{C})$ is the minimal $\sigma$-algebra containing the set $\mathscr{C}$ of all the cylinders with a finite dimensional basis, the couple $\left(\mathrm{R}^{3 \mathrm{~T}}, \mathscr{B}\left(\mathrm{R}^{3 \mathrm{~T}}\right)\right)$ will be the measurable space of all the trajectories of an arbitrary 
process, namely the trajectory space. On this trajectory space every process $\eta(t)$ induces a probability measure $P_{\eta}$ which is firstly defined on $\mathscr{C}$ by means of $P$, i.e.

$P_{\eta}\left(\mathrm{C}_{\tau}(a)\right)=P\left(\boldsymbol{\eta}\left(t_{1}\right) \in \mathrm{A}_{1}, \ldots, \boldsymbol{\eta}\left(r_{n}\right) \in \mathrm{A}_{n}\right)$,

and then extended, by means of additivity and continuity, to $\mathscr{B}\left(\mathrm{R}^{3 \mathrm{~T}}\right)$. Of course every different process will define a different probability on the same trajectory space. For instance the Wiener process $\beta(t)$ of $(18)$, characterized by a transition probability density

$P_{\mathrm{W}}(\boldsymbol{r}+\Delta \boldsymbol{r}, t+\Delta t ; \boldsymbol{r}, t)=(4 \pi \nu \Delta t)^{-3 / 2} \exp \left[-(\Delta \boldsymbol{r})^{2} / 4 \nu \Delta t\right]$ for $\Delta t>0$,

defines on $\left(\mathrm{R}^{3 \mathrm{~T}} ; \not{B}\left(\mathrm{R}^{3 \mathrm{~T}}\right)\right)$ the well-known Wiener measure $P_{\mathrm{w}}$ by extension of the following conditional probabilities on $\mathscr{C}$ :

$P_{\mathrm{W}}\left(\mathrm{C}_{\tau}(\boldsymbol{a}) \mid \boldsymbol{\beta}\left(t_{\mathrm{i}}\right)=\boldsymbol{r}_{\mathrm{i}}\right)=\int_{\mathbf{A}_{1}} \ldots \int_{\mathrm{A}_{n}} P_{\mathrm{W}}\left(\boldsymbol{r}_{1}, t_{1} ; \boldsymbol{r}_{\mathrm{i}}, t_{\mathrm{i}}\right) P_{\mathrm{W}}\left(\boldsymbol{r}_{2}, t_{2} ; \boldsymbol{r}_{1}, t_{1}\right) \ldots P_{\mathrm{W}}\left(\boldsymbol{r}_{n}, t_{n} ; \boldsymbol{r}_{n-1}, r_{n-1}\right) \mathrm{d}^{3} \boldsymbol{r}_{1} \ldots \mathrm{d}^{3} \boldsymbol{r}_{n}$.

The other measures that we will consider are defined by the solutions $\xi(t)$ of $(18)$ with the initial condition (21). Of course, for every different $\psi$, or $\boldsymbol{v}_{(+)}$, we will get a different process and hence a different probability $P_{\xi}\left(\mid \boldsymbol{\xi}_{\mathrm{i}}\right)$ on the trajectory space. In particular the process $\boldsymbol{\xi}_{0}(t)=\boldsymbol{\xi}_{\mathrm{i}}+\boldsymbol{\beta}(t)-\boldsymbol{\beta}\left(t_{\mathrm{i}}\right)$, corresponding to a $\boldsymbol{v}_{(+)}$identically zero, will define a conditional probability coincident with the Wiener measure $P_{\mathrm{w}}(\mid \xi)$. Moreover all these probability measures are connected: If $P_{1}$ and $P_{2}$ are defined by the solutions $\xi_{1}(t)$ and $\xi_{2}(t)$ of $(18)$ corresponding to two different $v_{(+)}^{(1)}$, and $v_{+}^{(2)}$, but to the same initial condition, a theorem due to Girsanov states that $P_{2}$ is absolutely continuous with respect to $P_{1}$ and that, if $\delta v_{(+)}=v(2)-v\left(\begin{array}{l}1) \\ +\end{array}\right)$, the corresponding Radon derivative takes the form

$\frac{\mathrm{d} P_{2}}{\mathrm{~d} P_{1}}\left[\boldsymbol{\xi}_{1}()\right]=\exp \left(U\left[\xi_{1}()\right]\right)$,

where the functional $U$ is defined by an Ito stochastic integral:

$U\left[\xi_{0}()\right]=\frac{1}{2 \nu} \int_{t_{\mathrm{i}}}^{t_{\mathrm{f}}} \delta \boldsymbol{v}_{(+)}(\boldsymbol{\xi}(t), t) \cdot \mathrm{d} \boldsymbol{\beta}(t)-\frac{1}{4 \nu} \int_{t_{\mathrm{i}}}^{t_{\mathrm{f}}}\left[\delta \boldsymbol{v}_{(+)}\left(\boldsymbol{\xi}_{1}(t), t\right)\right]^{2} \mathrm{~d} t$.

When in particular $\boldsymbol{\xi}_{1}^{(t)}=\boldsymbol{\xi}_{0}(t)$ we have

$U\left[\xi_{0}(\quad)\right]=\frac{1}{2 \nu} \int_{t_{\mathrm{i}}}^{t_{\mathrm{f}}} \boldsymbol{v}_{(+)}\left(\boldsymbol{\xi}_{0}(t), t\right) \cdot \mathrm{d} \boldsymbol{\beta}(t)-\frac{1}{4 \nu} \int_{t_{\mathrm{i}}}^{t_{\mathrm{f}}}\left[\boldsymbol{v}_{(+)}\left(\boldsymbol{\xi}_{0}(t), t\right)\right]^{2} \mathrm{~d} t$

and the expectation value of an arbitrary random functional $F[\xi(\quad)]$ can always be calculated by means of the Wiener measure induced on the trajectory space by $\xi_{0}(t)$ : i.e.

$E\left(F[\boldsymbol{\xi}()] \mid \boldsymbol{\xi}_{\mathrm{i}}\right)=E\left(F\left[\boldsymbol{\xi}_{0}()\right] \mathrm{e}^{U\left(\boldsymbol{\xi}_{0}()\right)} \mid \boldsymbol{\xi}_{\mathrm{i}}\right)$.

When $v_{(+)}$is a gradient, as shown in (19) for stochastic mechanics, the Ito differential formula and the relations (22) and (23) imply a further simplification of (29) containing no Ito integrals [10]:

$U\left(\boldsymbol{\xi}_{0}()\right)=W_{+}\left(\xi_{0}\left(t_{\mathrm{f}}\right), t_{\mathrm{f}}\right)-W_{(+)}\left(\xi_{0}\left(t_{\mathrm{i}}\right), t_{\mathrm{i}}\right)+\frac{1}{\hbar} \int_{t_{\mathrm{i}}}^{t_{\mathrm{f}}}\left(\frac{\hbar^{2}}{m} \frac{\nabla^{2} R}{R}-V\right)\left(\boldsymbol{\xi}_{0}(t), t\right) \mathrm{d} t$.

It is possible now to derive, from this classical probabilistic formulation, a path integral expression for the transition probability densities of the stochastic mechanics. If 


$$
\begin{aligned}
J_{\mathrm{A}}(r() ; t) & =1, & r() \in \mathrm{C}_{t}(\mathrm{~A}) ; \\
& =0, & \boldsymbol{r}() \notin \mathrm{C}_{t}(\mathrm{~A}) ;
\end{aligned}
$$

$$
t \in\left[t_{\mathrm{i}}, t_{\mathrm{f}}\right), \mathrm{A} \in \mathscr{B}\left(\mathbb{R}^{3}\right),
$$

is the indicator functional of the one-dimensional cylinder $C_{t}(\mathrm{~A})$ in $\mathrm{R}^{3 \mathrm{~T}}$, from (30) we have $\int_{\mathrm{A}} P\left(\boldsymbol{r}_{\mathrm{f}}, t_{\mathrm{f}} ; \boldsymbol{r}_{\mathrm{i}}, t_{\mathrm{i}}\right) \mathrm{d}^{3} \boldsymbol{r}_{\mathrm{f}}=P\left(\boldsymbol{\xi}\left(t_{\mathrm{f}}\right) \in \mathrm{A} \mid \xi_{\mathrm{i}}=\boldsymbol{r}_{\mathrm{i}}\right)=E\left(J_{\mathrm{A}}\left[\xi() ; t_{\mathrm{f}}\right] \mid \xi_{\mathrm{i}}=r_{\mathrm{i}}\right)=E\left(J_{\mathrm{A}}\left[\xi_{0}() ; t_{\mathrm{f}}\right] \mathrm{e}^{U\left(\xi_{0}()\right)} \mid \xi_{\mathrm{i}}=r_{\mathrm{i}}\right)$

We can now calculate successive approximations of this probability by means of a succession of partitions $\left\{t_{0}\right.$, $\left.t_{1}, \ldots, t_{n}\right\}, n \in \mathbb{N}$, of $\left[t_{\mathrm{i}}, t_{\mathrm{f}}\right]$, with $t_{0}=t_{\mathrm{i}}, t_{n}=t_{\mathrm{f}}$ and

$t_{K+1}-t_{K}=\frac{t_{\mathrm{f}}-t_{\mathrm{i}}}{n}=\Delta t>0, \quad K=0,1, \ldots, n-1, n$,

and the corresponding restrictions of the Wiener measure to $C_{\eta}(a), \tau=\left\{t_{1}, \ldots, t_{n}\right\}, a=\mathbb{R}^{3} \times \ldots \times \mathbb{R}^{3} \times A=\mathbb{R}^{3 n} \times A$, so that we have

$$
\begin{aligned}
& \int_{\mathrm{A}} P\left(\boldsymbol{r}_{\mathrm{f}}, r_{\mathrm{f}} ; \boldsymbol{r}_{\mathrm{i}}, t_{\mathrm{i}}\right) \mathrm{d}^{3} \boldsymbol{r}_{\mathrm{f}} \sim \int_{\mathbb{R}^{3}} P_{\mathrm{W}}\left(r_{1}, t_{1} ; \boldsymbol{r}_{\mathrm{i}}, t_{\mathrm{i}}\right) \mathrm{d}^{3} \boldsymbol{r}_{1} \ldots \int_{\mathrm{R}^{3}} P_{\mathrm{W}}\left(\boldsymbol{r}_{n-1}, t_{n-1} ; \boldsymbol{r}_{n-2}, t_{n-2}\right) \mathrm{d}^{3} \boldsymbol{r}_{n-1} \\
& \quad \times \int_{\mathrm{A}} P_{\mathrm{W}}\left(\boldsymbol{r}_{\mathrm{f}}, t_{\mathrm{f}} ; \boldsymbol{r}_{n-1}, t_{n-1}\right) \mathrm{d}^{3} \boldsymbol{r}_{\mathrm{f}} \exp \left[U^{(n)}\left(\boldsymbol{r}_{\mathrm{i}}, \boldsymbol{r}_{1}, \ldots, \boldsymbol{r}_{\mathrm{f}}\right)\right]=\int_{\mathrm{A}} P^{(n)}\left(\boldsymbol{r}_{\mathrm{f}}, t_{\mathrm{f}} ; \boldsymbol{r}_{\mathrm{i}}, t_{\mathrm{i}}\right) \mathrm{d}^{3} \boldsymbol{r}_{\mathrm{f}},
\end{aligned}
$$

where

$$
U^{(n)}\left(r_{\mathrm{i}}, r_{1}, \ldots, r_{\mathrm{f}}\right)=\frac{1}{2 \nu} \sum_{K=0}^{n-1} v_{(+)}\left(r_{K}, t_{K}\right) \Delta r_{K}-\frac{1}{4 \nu} \sum_{K=0}^{n-1}\left[v_{(+)}\left(r_{K}, t_{K}\right)\right]^{2} \Delta t
$$

is the approximation to $U[r()]$ calculated along a path such that $r\left(t_{K}\right)=r_{K} ; K=0,1, \ldots, n\left(r_{\mathrm{i}}=r_{0}, r_{\mathrm{f}}=r_{n}\right)$, and $\Delta \boldsymbol{r}_{K}=\boldsymbol{r}_{K+1}-\boldsymbol{r}_{K}$. Now, from (26) and (32) we have

$$
P^{(n)}\left(r_{\mathrm{f}}, t_{\mathrm{f}} ; \boldsymbol{r}_{\mathrm{i}}, t_{\mathrm{i}}\right)=\int_{\mathbb{R}^{3}} \mathrm{~d}^{3} \boldsymbol{r}_{1} \ldots \int_{\mathbb{R}^{3}} \mathrm{~d}^{3} \boldsymbol{r}_{n-1}(4 \pi \nu \Delta t)^{-3 n / 2} \exp \left(-\frac{1}{4 \nu \Delta t} \sum_{K=0}^{n-1}\left[\Delta \boldsymbol{r}_{K}-\boldsymbol{v}_{(+)}\left(\boldsymbol{r}_{K}, t_{\mathrm{K}}\right) \Delta t\right]^{2}\right) .
$$

When eventually $n \rightarrow \infty$, we get $P^{(n)} \rightarrow P$ and this statement is condensed in the following path integral,

$$
\left.P\left(r_{\mathrm{f}}, t_{\mathrm{f}} ; \boldsymbol{r}_{\mathrm{i}}, t_{\mathrm{i}}\right)=\int_{\mathrm{i} \rightarrow \mathrm{f}} \exp \left[-\frac{1}{4 \nu}\left(\frac{\mathrm{d} \boldsymbol{r}(t)}{\mathrm{d} t}-\boldsymbol{v}_{(+)}(\boldsymbol{r}(t), t)\right)^{2} \mathrm{~d} t\right] \mathrm{D}^{\prime}[\boldsymbol{r}())\right],
$$

whose exact meaning is the fact that $P$ is the limit of (33) where $n \rightarrow \infty$. The expression (34), derived in a number of papers [9] in a different way, despite a formal analogy with (17), is a true probabilistic statement, as the former derivation shows. Moreover a comparison of (17) and (34) shows also that, as remarked before, while $K$ is determined only by the dynamics of the system, $P$ depends on the state through the form of $v_{(+)}$.

In conclusion we can remark that the existence of quantum interferences can no more be seen as an absolute obstacle to the existence of particle trajectories. This, of course, could be expected since stochastic mechanics simulates the quantum behaviour, but is also essentially based on the idea of trajectories in space and time. In fact we have shown explicitly that it is possible to calculate suitable real positive transition probabilities that can be added together to have the interference pattern. It can thus be said that in a wave function $\psi$ there is more information than that which can be calculated by means of the usual quantum formulas. For example in (34) we were able to calculate $P\left(r_{\mathrm{f}}, t_{\mathrm{f}}, \boldsymbol{r}_{\mathrm{i}}, t_{\mathrm{i}}\right)$ in a general situation where no quantum mechanical analogs 
exist. The particular meaning of this result for the concept of conditional probability in quantum mechanics will be discussed in a subsequent paper.

The author wants to thank Professors J.P. Vigier and F. Guerra for enlightening discussions and suggestions. He also wants to thank the Italian INFN for a grant which made this research possible.

\section{References}

[1] G. Baburek, H. Rauch and D. Tuppinger, Phys. Rev. A 34 (1986) 2600.

[2] D. Bohm, Phys. Rev. 85 (1952) 166, 180;

D. Bohm and J.P. Vigier, Phys. Rev. 96 (1954) 208.

[3] E. Nelson, Dynamical theories of Brownian motion (Princeton Univ. Press, Princeton, 1967); Quantum fluctuations (Princeton Univ. Press, Princeton, 1985);

N. Cufaro-Petroni, H. Raffii-Tabar and J.P. Vigier, Brownian and quantum motions based on the reality of random paths in physical space and time, Nature, in press;

H. Raffii-Tabar, Phys. Lett. A 138 (1989) 353.

[4] R.P. Feynman, Rev. Mod. Phys. 20 (1948) 267;

R.P. Feynman and A.R. Hibbs, Quantum mechanics and path integrals (McGraw-Hill, New York, 1965).

[5] F. Guerra and L.M. Morato, Phys. Rev. D 27 (1983) 1774;

F. Guerra and R. Marra, Phys. Rev. D 28 (1983) 1916; 29 (1984) 1647

[6] W.H. Fleming and R. Rishel, Deterministic and stochastic optimal control (Springer, Berlin, 1975).

[7 ] I.I. Gihman and A.V. Skorohod, Stochastic differential equations (Springer, Berlin, 1972); Introduction à la théorie des processus aléatoires (Mir, Moscow, 1980).

[8] A.N. Shiryayev, Probability (Springer, Berlin, 1984).

[9] F.W. Wiegel, Physica 33 (1967) 734; 37 (1967) 105;

M.S. Wang; Phys. Rev. A 38 (1988) 5401.

[10] F. Guerra, Meccanica stochastica (Rome, 1986), preprint; Equazione differenziali stocastiche (Rome, 1986), preprint. 